\title{
Latency and Selectivity of Single Neurons Indicate Hierarchical Processing in the Human Medial Temporal Lobe
}

\author{
Florian Mormann, ${ }^{1,2}$ Simon Kornblith, ${ }^{1}$ Rodrigo Quian Quiroga, ${ }^{1,3}$ Alexander Kraskov, ${ }^{1,4}$ Moran Cerf, ${ }^{1,2}$ Itzhak Fried, ${ }^{2,5,6}$ \\ and Christof Koch ${ }^{1}$ \\ ${ }^{1}$ Division of Biology, California Institute of Technology, Pasadena, California 91125, ${ }^{2}$ Department of Neurosurgery, University of California, Los Angeles, \\ Los Angeles, California 90095, ${ }^{3}$ Department of Engineering, University of Leicester, Leicester LE1 7RH, United Kingdom, ${ }^{4}$ Institute of Neurology, University \\ College London, London WC1N 3BG, United Kingdom, ${ }^{5}$ Functional Neurosurgery Unit, Tel-Aviv Sourasky Medical Center, Tel-Aviv 64239, Israel, and \\ ${ }^{6}$ Sackler Faculty of Medicine, Tel-Aviv University, Tel-Aviv 69978, Israel
}

Neurons in the temporal lobe of both monkeys and humans show selective responses to classes of visual stimuli and even to specific individuals. In this study, we investigate the latency and selectivity of visually responsive neurons recorded from microelectrodes in the parahippocampal cortex, entorhinal cortex, hippocampus, and amygdala of human subjects during a visual object presentation task. During 96 experimental sessions in 35 subjects, we recorded from a total of 3278 neurons. Of these units, 398 responded selectively to one or more of the presented stimuli. Mean response latencies were substantially larger than those reported in monkeys. We observed a highly significant correlation between the latency and the selectivity of these neurons: the longer the latency the greater the selectivity. Particularly, parahippocampal neurons were found to respond significantly earlier and less selectively than those in the other three regions. Regional analysis showed significant correlations between latency and selectivity within the parahippocampal cortex, entorhinal cortex, and hippocampus, but not within the amygdala. The later and more selective responses tended to be generated by cells with sparse baseline firing rates and vice versa. Our results provide direct evidence for hierarchical processing of sensory information at the interface between the visual pathway and the limbic system, by which increasingly refined and specific representations of stimulus identity are generated over time along the anatomic pathways of the medial temporal lobe.

Key words: single-unit recordings; object recognition; parahippocampal gyrus; entorhinal cortex; hippocampus; amygdala

\begin{abstract}
Introduction
The inferior and medial regions of the temporal lobe in human and nonhuman primates comprise the distal stages of the ventral visual pathway and parts of the limbic system, responsible for encoding and retrieval of mnemonic information. The differential contribution of these regions to the processing and elaboration of information at the interface between perception and memory remains an open question (Squire et al., 2004). Recordings of single-neuron activity in monkey visual temporal cortex led to the discovery of neurons that respond selectively to certain categories of stimuli such as faces or objects (cf. Logothetis and Sheinberg, 1996; Tanaka, 1996; Freedman and Miller, 2008). Recordings of single-cell activity in the human medial temporal lobe
\end{abstract}

\footnotetext{
Received April 15, 2008; revised June 23, 2008; accepted July 12, 2008.

This work was supported by the European Commission (Marie Curie Outgoing International Fellowship 040445), National Institute of Neurological Disorders and Stroke, Defense Advanced Research Projects Agency, the Engineering and Physical Science Research Council, and the Mathers Foundation. We thank all patients for their participation, and Eric Behnke, Tony Fields, Emily Ho, Eve Isham, Kelsey Laird, Neel Parikshak, and Anna Postolova for technical assistance.

Correspondence should be addressed to Dr. Florian Mormann, California Institute of Technology, Division of Biology, MS 216-76, 1200 East California Boulevard, Pasadena, CA 91125. E-mail: fmormann@yahoo.de. D0I:10.1523/JNEUROSCl.1640-08.2008

Copyright $\odot 2008$ Society for Neuroscience $\quad 0270-6474 / 08 / 288865-08 \$ 15.00 / 0$
}

(MTL) have revealed similar category neurons (Fried et al., 1997; Kreiman et al., 2000) and even neurons that show selective and invariant responses to different pictures of an individual, including their written name (Quiroga et al., 2005). Neuroanatomical studies in monkeys have identified direct connections between different regions of the inferior and medial temporal lobe $(\mathrm{Su}-$ zuki, 1996). Whereas visual response latencies to different types of stimuli have been reported for different temporal areas in monkeys (Table 1), few studies provide a direct regional comparison (Leonard et al., 1985; Liu and Richmond, 2000; Naya et al., 2001, 2003), and reports on human latency data to date remain elusive. We, here, systematically investigate the latency and selectivity of visually responsive neurons and report evidence for hierarchical processing of visual stimuli in the human MTL.

\section{Materials and Methods}

All studies conformed to the guidelines of the Medical Institutional Review Board at the University of California, Los Angeles. Electrode locations were based exclusively on clinical criteria and were verified by magnetic resonance imaging (MRI) or by computer tomography coregistered to preoperative MRI. Each electrode probe had nine microwires protruding from its tip, eight high-impedance recording channels (typically 200-400 $\mathrm{k} \Omega$ ), and one low-impedance reference with stripped insulation. The differential signal from the microwires 
Table 1. Selected studies reporting visual response latencies of neurons in the temporal lobe of macaque monkeys

\begin{tabular}{|c|c|c|c|}
\hline Reference & Region & Stimuli & Latency \\
\hline Gross et al., 1972 & IT & Colors/shapes & NS \\
\hline Fuster and Jervey, 1982 & IT & Colors & $\sim 100 \mathrm{~ms}$ \\
\hline Perrett et al., 1982 & IT & Face/nonface & $80-160 \mathrm{~ms}$ \\
\hline Desimone et al., 1984 & IT & Face/hand & NS \\
\hline Baylis et al., 1987 & IT & Face/nonface & $\sim 100 \mathrm{~ms}$ \\
\hline \multirow[t]{3}{*}{ Fuster, 1990} & IT & Colors & $270 \mathrm{~ms}$ \\
\hline & & Symbols & $203 \mathrm{~ms}$ \\
\hline & & Unselective & $159 \mathrm{~ms}$ \\
\hline Li et al., 1993 & IT & Faces/objects/patterns & $\sim 70 \mathrm{~ms}$ \\
\hline Sheinberg and Logothetis, 2001 & IT & Objects/in natural scenes & $\sim 100 \mathrm{~ms}$ \\
\hline Tamura and Tanaka, 2001 & $\mathrm{IT}(\mathrm{TE})$ & Faces/animals/objects/shapes & $121.0 \pm 57.8 \mathrm{~ms}$ \\
\hline Hung et al., 2005 & IT & Objects & 125 ms best \\
\hline Hasselmo et al., 1989 & STS/IT & Faces & $\sim 100 \mathrm{~ms}$ \\
\hline Tovee et al., 1994 & STS/IT & Primate and human faces & 70-90 ms (typically) \\
\hline \multirow[t]{2}{*}{ Eifuku et al., 2004} & STS & Faces & $91-120 \mathrm{~ms}$ \\
\hline & IT & Faces & $117-198 \mathrm{~ms}$ \\
\hline \multirow[t]{2}{*}{ Kiani et al., 2005} & STS/IT & Human faces & $103 \mathrm{~ms}$ \\
\hline & & Non-primate faces & $118 \mathrm{~ms}$ \\
\hline Bruce et al., 1981 & STS & Shapes/faces & $140 \mathrm{~ms}$ (200-300 to faces) \\
\hline Perrett et al., 1984 & STS & Faces & $135 \mathrm{~ms}$ \\
\hline Perrett et al., 1988 & STS & Faces/jumbled faces & NS \\
\hline Keysers et al., 2001 & STS & Faces/nonfaces & 108 ms (56-171) \\
\hline Oram and Perrett, 1992 & STS, TPO, PGa & Heads/objects & 119 ms (69-213) \\
\hline Wilson et al., 1990 & Hipp, rhinal, PHC & Shapes & NS \\
\hline Riches et al., 1991 & Hipp, EC, PHC & Shapes & NS \\
\hline Fahy et al., 1993 & EC, PRh, IT & Complex pictures & NS (1 EC, $150 \mathrm{~ms})$ \\
\hline \multirow[t]{4}{*}{ Xiang and Brown, 1998} & IT (TE) & Novelty/recency/familiarity & 75 ms (differential) \\
\hline & PRh & & 105 ms (differential) \\
\hline & $\mathrm{EC}$ & & 135 ms (differential) \\
\hline & & & 105 ms, 165 ms (category) \\
\hline Xiang and Brown, 1999 & Hipp, EC, IT & Shapes & 195 ms (individual) \\
\hline \multirow[t]{2}{*}{ Liu and Richmond, 2000} & IT & Patterns & 78 ms (iqr 60 -115) \\
\hline & PRh & & 144 ms (iqr 109-185) \\
\hline \multirow[t]{2}{*}{ Naya et al., 2001} & IT (TE) & Shapes & $77 \mathrm{~ms}$ \\
\hline & $\operatorname{PRh}(\mathrm{A36})$ & & $89 \mathrm{~ms}$ \\
\hline \multirow[t]{2}{*}{ Naya et al., 2003} & IT (TE) & Shapes (Fourier descriptors) & $86.2 \pm 1.5 \mathrm{~ms}$ \\
\hline & PRh (A36) & & $93.8 \pm 3.2 \mathrm{~ms}$ \\
\hline Nakamura et al., 1994 & Temporal pole & Faces/nonfaces & $100-200$ ms (mode 150) \\
\hline Suzuki et al., 1997 & $\mathrm{EC}$ & Objects & $181 \mathrm{~ms}(100-300)$ \\
\hline Watanabe and Niki, 1985 & Нipp & Cue light & $120-180$ ms (mostly $120-140)$ \\
\hline \multirow[t]{2}{*}{ Rolls et al., 1989} & Нipp & Shapes & 168 ms (100-200) \\
\hline & & & $196 \mathrm{~ms}$ \\
\hline Rolls et al., 1993 & Hipp & Shapes/TV scenes & $140-260 \mathrm{~ms}$ \\
\hline Eifuku et al., 1995 & Hipp & Objects in places & $209 \pm 9 \mathrm{~ms}$ \\
\hline \multirow[t]{2}{*}{ Yanike et al., 2004} & Hipp & Location/scene & Familiar: $152 \pm 9 \mathrm{~ms}$ \\
\hline & & & New: $152 \pm 10 \mathrm{~ms}$ \\
\hline Rolls et al., 2005 & Hipp & Shapes/objects & 3 reported: $120,150,180 \mathrm{~ms}$ \\
\hline Sanghera et al., 1979 & Amy & Faces/objects & $100-180$ ms (mostly $110-130)$ \\
\hline \multirow[t]{2}{*}{ Leonard et al., 1985} & Amy & Faces & $110-200 \mathrm{~ms}$ \\
\hline & STS & Faces & $90-140 \mathrm{~ms}$ \\
\hline Nakamura et al., 1992 & Amy & Faces/nonfaces & $60-300 \mathrm{~ms}(167 \pm 51)$ \\
\hline Gothard et al., 2007 & Amy & Faces/nonfaces & $110-140 \mathrm{~ms}$ \\
\hline
\end{tabular}

IT, Inferotemporal cortex; STS, superior temporal sulcus; PHC, parahippocampal cortex; PRh, perirhinal cortex; EC, enthorhinal cortex; Amy, amygdala; Hipp, hippocampus; iqr, interquartile range; differential, differential responses distinguishing old from new items; NS, not specified.

was amplified using a 64-channel Neuralynx system, filtered between 1 and $9000 \mathrm{~Hz}$, and sampled at $28 \mathrm{kHz}$. Spike detection and sorting was performed after bandpass filtering the signals between 300 and $3000 \mathrm{~Hz}$ (Quiroga et al., 2004).

Each recording session lasted $\sim 30 \mathrm{~min}$. Subjects were sitting in bed, facing a laptop computer on which pictures of famous individuals, landmarks, animals, or objects were shown. A median number of 97 (range, 60-202) different images were shown per session, centered on a laptop screen and covering $\sim 1.5^{\circ}$, and displayed six times each for $1 \mathrm{~s}$ in pseudorandom order (Quiroga et al., 2005). After image offset, subjects had to indicate whether the picture contained a human face or something else by pressing the " $\mathrm{Y}$ " and " $\mathrm{N}$ " keys, respectively. This simple task, on which performance was virtually flawless, required them to attend to the pictures. Every stimulus presentation was preceded by a fixation cross for $500 \mathrm{~ms}$ to assess baseline firing activity. In a slightly different variant of the paradigm (23 of 96 sessions), images were presented for $500 \mathrm{~ms}$ ( 20 sessions) or $750 \mathrm{~ms}$ ( 3 sessions), and the attention task was omitted. Absence of a significant influence of the presentation time on the observed response latencies was confirmed post hoc by nonparametric one-way ANOVA (Kruskal-Wallis; $p=0.18)$.

To determine whether a unit responded selectively to one or more of 

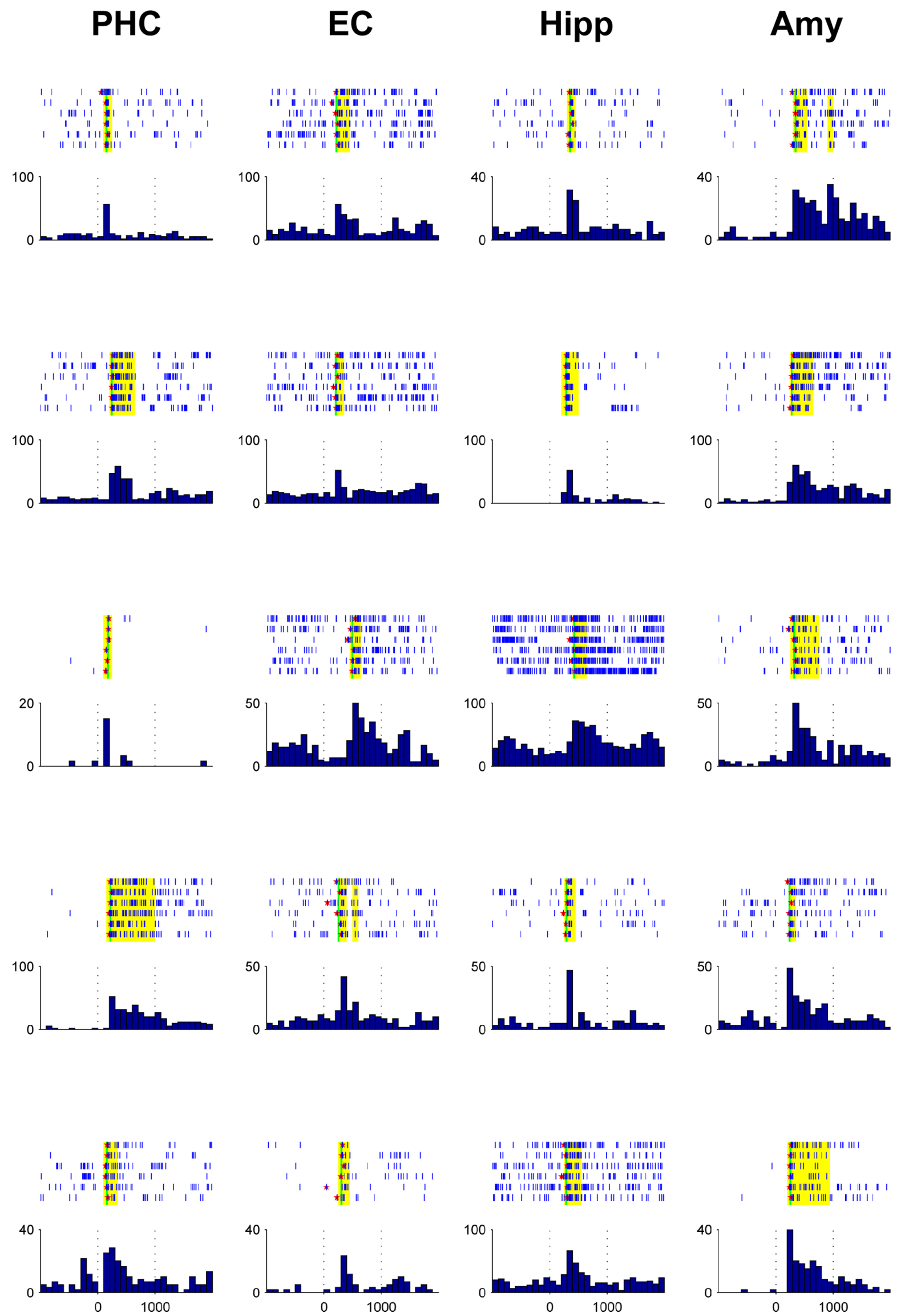

Figure 1. Response plots of 20 exemplary neurons in the parahippocampal cortex (PHC), entorhinal cortex (EC), hippocampus (Hipp), and amygdala (Amy). Displayed are spike raster plots for the six stimulus presentations and the peristimulus time histogram (firing rate in hertz vs time in milliseconds) for each response. Time bins matching the criterion for a response are marked in yellow. Spike train onsets as determined by Poisson spike train analysis are denoted as red stars and their median latency by a vertical green line. 
$\mathrm{PHC}$
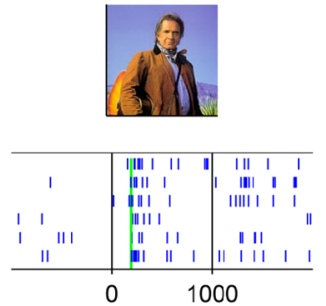

PHC (N=47)

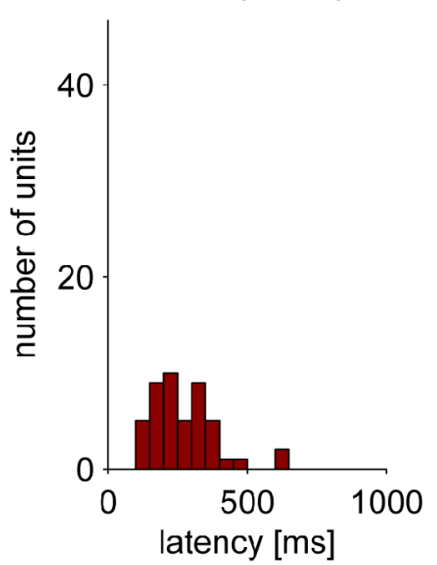

EC
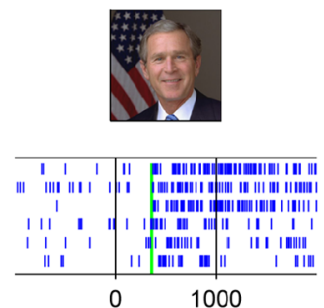

Hipp
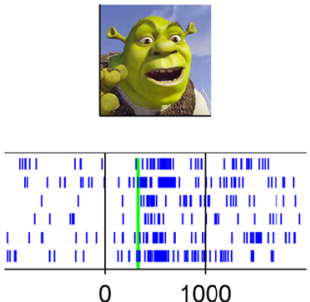

Amy
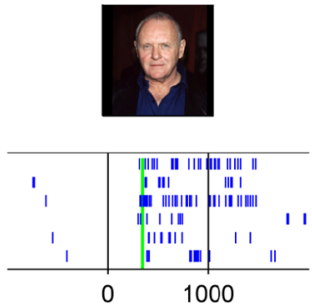

$E C(N=79)$

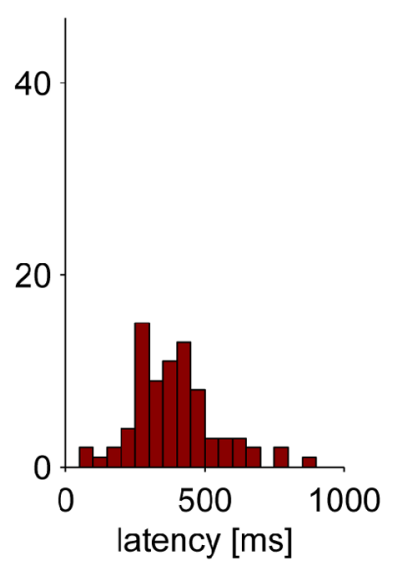

Hipp ( $\mathbf{N}=171)$

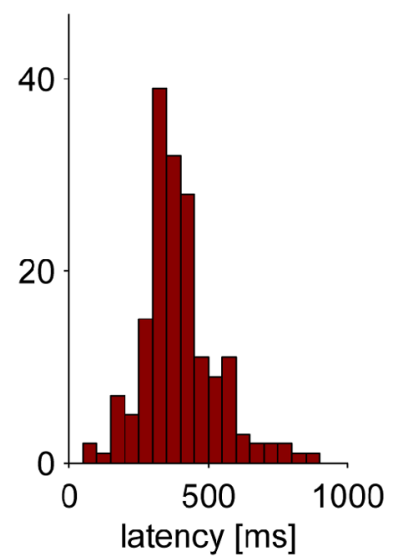

Amy $(\mathrm{N}=101)$

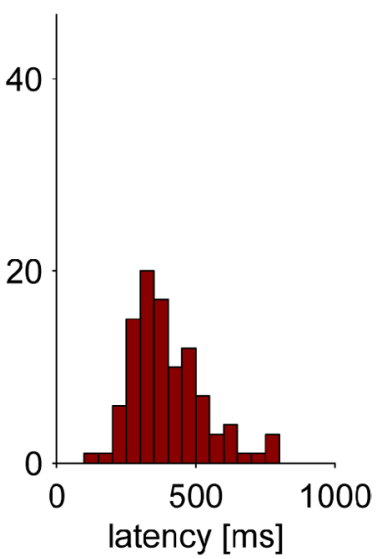

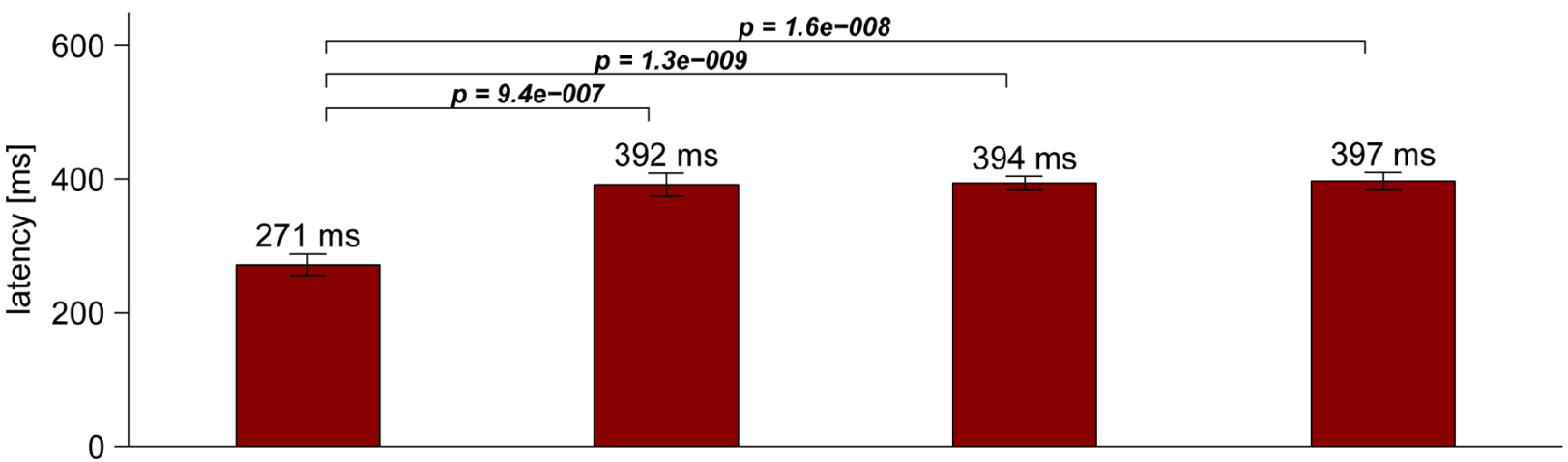

Figure 2. Response latency of neurons in the human MTL. A total of 398 neurons responded to one or more visual stimuli with a significant $(p<0.001)$ increase in firing rate relative to baseline. Top, Exemplary raster plots of visual responses in the parahippocampal cortex (PHC), entorhinal cortex (EC), hippocampus (Hipp), and amygdala (Amy), with typical latencies calculated using Poisson spike train analysis and denoted by green vertical lines. Middle, Latency histograms of responsive neurons in each region (in $50 \mathrm{~ms}$ bins, absolute cell numbers). Bottom, Mean latencies \pm SEM and pairwise statistical comparison of latencies across regions (two-sided Mann-Whitney $U$ test; only $p$ values $<0.05$ are shown).

the stimuli presented, we divided the $1000 \mathrm{~ms}$ after stimulus onset into 19 overlapping $100 \mathrm{~ms}$ bins, and for each bin we compared the spike rates for the six presentations of each stimulus to the baseline intervals of 500 $\mathrm{ms}$ before all of the stimulus onsets in a session $(\sim 100 \times 6)$ by means of a two-tailed Mann-Whitney $U$ test, using the Simes procedure (Rodland, 2006) to correct for multiple comparisons and applying a conservative significance threshold of $p=0.001$ to reduce false-positive detections. Only responsive units were included in the subsequent latency and selectivity analyses.

Onset latencies for responsive units were determined by Poisson spike train analysis (Hanes et al., 1995). For this procedure, the interspike intervals (ISIs) of a given unit are processed continuously over the entire recording session, and the onset of a spike train is detected based on its deviation from a baseline Poisson, i.e., exponential, distribution of ISIs (regardless of the experimental paradigm). For each response-eliciting stimulus, we determined the time between stimulus onset and the onset of the first spike train in all six presentations. Only spike train onsets within the first $1000 \mathrm{~ms}$ after stimulus onset were considered. The median length of these six time intervals was taken as response latency. For sparsely firing units with mean baseline firing activity of $<2 \mathrm{~Hz}$, Poisson spike train analysis generally failed to pick up any onset spike, thus we used the median latency of the first spike during stimulus presentation instead. To minimize spurious latency values, we excluded responses for which the onsets of the three trials closest to the calculated response latency were $>200 \mathrm{~ms}$ apart. For a neuron responding to more than one stimulus, the median of the different stimulus latencies was taken.

For the nonparametric correlation analysis, selectivity of each unit was operationally defined as the reciprocal value of the relative number of response-eliciting stimuli.

Baseline firing rates of the responsive cells were calculated from the $500 \mathrm{~ms}$ before stimulus onset and quantified as the median across six presentations. For a neuron responding to more than one stimulus, the median of the baseline rates for different stimuli was taken. 


\section{Results}

During 96 sessions, we recorded from 3278 neurons (1356 multi units, 1922 single units) in 35 subjects with pharmacologically intractable epilepsy (29 right handed, 20 male, 17-54 years old), implanted with chronic electrodes to localize the seizure focus for possible surgical resection (Fried et al., 1997). We report data from microelectrode recordings in the hippocampus, amygdala, entorhinal cortex, and parahippocampal cortex [in the part of the parahippocampal gyrus that is posterior to the entorhinal and perirhinal cortex (cf. Insausti et al., 1998)]. Each recording session lasted $\sim 30 \mathrm{~min}$. Subjects were sitting in bed, facing a laptop computer on which $\sim 100$ pictures per session of different famous individuals, landmarks, animals, or objects were displayed for $1 \mathrm{~s}$ each, with six repetitions in pseudorandom order. Onset latencies for responsive units (i.e., units showing a significant increase in firing rate relative to baseline) were determined by Poisson spike train analysis (Hanes et al., 1995). Examples of responses from five different neurons in each MTL region are displayed in Figure 1.

A total of 398 units [47 of 293 (16\%) in the parahippocampal cortex; 79 of 844 (9\%) in the entorhinal cortex; 171 of 1194 (14\%) in the hippocampus; 101 of 947 (11\%) in the amygdala] responded significantly to one or more of the presented stimuli (cf. Waydo et al., 2006). Response latencies of these neurons yielded unimodal, localized distributions in all four regions (Fig. 2, top, middle). Average response latencies in the parahippocampal cortex $(271 \mathrm{~ms})$ were significantly earlier than those in the entorhinal cortex (392 ms), hippocampus (394 ms), and amygdala (397 ms), preceding these regions typically by $>100 \mathrm{~ms}$ [Figs. 2 (bottom), $4 A]$.

Because we used an automated, objective criterion to select responsive neurons and determine their response latencies, we cannot expect the specificity of our approach to be perfect, and the distributions in Figure 2 may thus be contaminated by a small percentage of spurious latencies, affecting especially the tails of the distributions. We identified the earliest reliable response latencies by visual inspection and found them to be $101 \mathrm{~ms}$ for the parahippocampal cortex, $206 \mathrm{~ms}$ for the entorhinal cortex, 204 $\mathrm{ms}$ for the hippocampus, and $220 \mathrm{~ms}$ for the amygdala.

Given the all-or-none character of the firing response (Quiroga et al., 2007), we evaluated response selectivity by the number of stimuli to which a neuron responded. Like the latency, selectivity varied across regions (Figs. 3, 4B). Whereas parahippocampal neurons responded on average to approximately five stimuli, neurons in the other three regions showed a significantly higher selectivity with an average of approximately two responseeliciting stimuli (Fig. 3, bottom). To rule out an influence of the total number of stimuli per session, the analysis was repeated after normalizing the number of response-eliciting stimuli by the total stimulus number, yielding analogous results (supplemental Fig. S1, available at www.jneurosci.org as supplemental material).

Analysis of baseline firing rates for the different MTL regions showed higher baseline activity for responsive neurons in the parahippocampal and entorhinal cortex than for hippocampal and amygdala neurons, but no prominent difference of the parahippocampal cortex from the other three regions as observed for latency and selectivity (Fig. 4C; supplemental Fig. S2, available at www.jneurosci.org as supplemental material).

Nonparametric correlation analysis across all 398 responsive neurons confirmed a highly significant direct relationship between latency and selectivity (Spearman's $\rho=0.24 ; p=9.5 \times$ $10^{-7}$ ). Separate regional analysis (Fig. 5) confirmed a statistically significant correlation between latency and selectivity in the parahippocampal cortex $(p=0.00009)$, entorhinal cortex $(p=$ $0.008)$, and hippocampus ( $p=0.038)$, but not in the amygdala $(p=0.495)$.

Both latency and selectivity, furthermore, showed a significant inverse correlation with the baseline firing rates across all 398 MTL neurons (Spearman's $\rho=-0.24, p=8.1 \times 10^{-7}$; Spearman's $\rho=-0.12, p=0.02$, respectively).

Finally, to rule out an influence of the underlying pathology of an epileptic brain, we repeated the entire analysis after excluding 65 cells (amounting to 16\%) that were located in the same brain hemisphere as the epileptic focus. All findings demonstrated in this study remained valid and significant, and mean response latencies changed by $<10 \mathrm{~ms}$ on average.

\section{Discussion}

The latencies found in the parahippocampal cortex, entorhinal cortex, and hippocampus reflect the well-established neuroanatomical connections of these structures, with the entorhinal cortex providing the predominant input to the hippocampus and receiving major connections from the parahippocampal region (Suzuki and Amaral, 1994). The finding that latencies of responsive amygdala neurons do not significantly differ from entorhinal and hippocampal latencies is consistent with neuroanatomical evidence that the amygdala has monosynaptic connections to the 


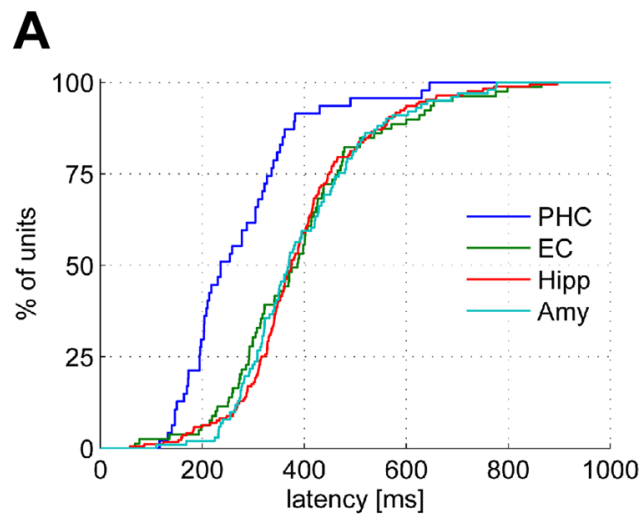

B

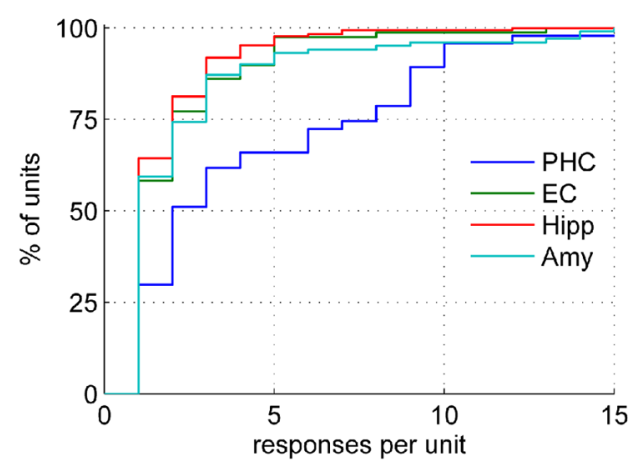

C

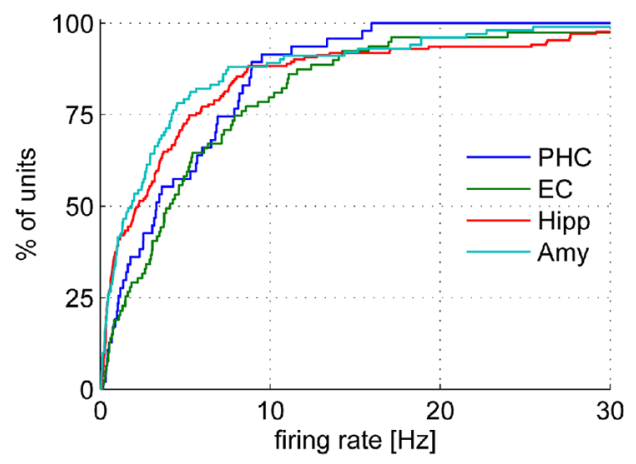

Figure 4. Cumulative distributions of response latencies $(\boldsymbol{A})$, selectivities as quantified by the number of responses per unit $(\boldsymbol{B})$, and baseline firing rates $(\boldsymbol{C})$ for the four MTL regions. PHC, Parahippocampal cortex; EC, entorhinal cortex; Hipp, hippocampus; Amy, amygdala.

entorhinal cortex and hippocampus (Suzuki, 1996; Pitkänen et al., 2002). Inputs to the amygdala originate from various sensory areas and other subcortical and cortical regions, among them the perirhinal cortex, which in turn receives parahippocampal inputs. Whereas some imaging studies have inferred a fast, subcortical sensory pathway to the amygdala (cf. Ohman et al., 2007), our results indicate that at least for the explicit, selective neural representations observed here, the processing time as reflected by the firing latency in the amygdala is comparable with entorhinal and hippocampal responses that are presumably generated along the ventral visual pathway.

The latencies observed by us are substantially larger than visual MTL latencies reported in monkeys. As can be seen from Table 1, mean latencies in the macaque entorhinal cortex, hippocampus, and amygdala range $\sim 150-200 \mathrm{~ms}$, with latencies in the perirhinal cortex being somewhat earlier but still significantly later than those in inferotemporal cortex (IT). To the best of our knowledge, no visual response latencies from neurons in the monkey parahippocampal cortex (area TH/TF) have been reported to date. Likewise, neuronal response latencies remain elusive for the ventral visual pathway in humans. Considering the data available, we can estimate the visual response latencies in the human MTL to be approximately twice as long as those observed in macaque monkeys. Based on this ratio, one could extrapolate the latencies in the human homolog of monkey IT [presumably the lateral occipital complex (cf. Grill-Spector and Malach, 2004)] to range in the vicinity of $200 \mathrm{~ms}$.

However, given the remarkable speed at which humans can discriminate stimulus categories (120 ms) (Kirchner and Thorpe, 2006), it is also conceivable that the latencies in monkey IT and its human homolog are not at all very different. In this case, the major difference would be the substantially longer delay between object recognition and the MTL latencies found by us. This in turn would suggest that IT responses in humans may undergo extensive further processing, possibly involving other regions, before reaching MTL and eliciting the highly selective responses observed here.

The visual tasks used in monkey MTL studies typically involve discrimination of novel versus familiar stimuli or association of different stimulus features. Recent electrophysiological studies in human subjects performing a learning task with initially unfamiliar stimuli likewise reported evidence for hippocampal and amygdala neurons that act as novelty or familiarity detectors without being stimulus specific (Rutishauser et al., 2006, 2008). It should be noted, however, that the responses described there are conceptually different from ours in that our stimulus material consists of images of single objects or persons that are already familiar to the subject and that no memory or association task is involved. Rather, these cells have been shown to encode with a high degree of invariance the category or identity of a presented object or person (Quiroga et al., 2005). A possible functional role of these neurons is to provide the link between perception and memory storage (Quiroga et al., 2008).

Remarkably, we find a prominent leap both in latency and selectivity between the parahippocampal cortex and its major projection area, the entorhinal cortex. Our data cannot unravel the detailed mechanisms of this hierarchical processing, but the involvement of various processes is conceivable. In the olfactory system of the locust, for instance, sparsening of representations is achieved by periodic feedforward inhibition (Perez-Orive et al., 2002). A similar mechanism could possibly be mediated by interneurons phase-locked to mediotemporal oscillations (Somogyi and Klausberger, 2005). Modulating influences could further arise through feedback loops between the parahippocampal cortex and inferotemporal and/or cingulate cortex, respectively (Suzuki and Amaral, 1994).

The direct relationship between latency and selectiveness of the visual responses observed here indicates a general mechanism of hierarchical processing (Grill-Spector and Malach, 2004; Freedman and Miller, 2008) by which increasingly refined and specific representations of stimulus identity are achieved over time along the anatomic pathways of the MTL (cf. Squire et al., 2004). A remarkable finding from our study is that this type of hierarchical processing occurs not only across different MTL regions, but also within regions such as the parahippocampal and entorhinal cortex and the hippocampus.

Interestingly, early and less-selective responses tended to be generated by cells with high baseline firing rates, whereas cells that responded later and more selectively tended to exhibit rather sparse baseline activity. Future technological advances may allow simultaneous recording of larger cell populations and thus pro- 
vide an opportunity to directly monitor the detailed mechanisms by which these cells implement the hierarchical processing described in this study.

\section{References}

Baylis GC, Rolls ET, Leonard CM (1987) Functional subdivisions of the temporal lobe neocortex. J Neurosci 7:330-342.

Bruce C, Desimone R, Gross CG (1981) Visual properties of neurons in a polysensory area in superior temporal sulcus of the macaque. J Neurophysiol 46:369-384.

Desimone R, Albright TD, Gross CG, Bruce C (1984) Stimulus-selective properties of inferior temporal neurons in the macaque. J Neurosci 4:2051-2062.

Eifuku S, Nishijo H, Kita T, Ono T (1995) Neuronal activity in the primate hippocampal formation during a conditional association task based on the subject's location. J Neurosci 15:4952-4969.

Eifuku S, De Souza WC, Tamura R, Nishijo H, Ono T (2004) Neuronal correlates of face identification in the monkey anterior temporal cortical areas. J Neurophysiol 91:358-371.

Fahy FL, Riches IP, Brown MW (1993) Neuronal activity related to visual recognition memory: long-term memory and the encoding of recency and familiarity information in the primate anterior and medial inferior temporal and rhinal cortex. Exp Brain Res 96:457-472.

Freedman DJ, Miller EK (2008) Neural mechanisms of visual categorization: insights from neurophysiology. Neurosci Biobehav Rev 32:311-329.

Fried I, MacDonald KA, Wilson CL (1997) Single neuron activity in human hippocampus and amygdala during recognition of faces and objects. Neuron 18:753-765.

Fuster JM (1990) Inferotemporal units in selective visual attention and short-term memory. J Neurophysiol 64:681-697.

Fuster JM, Jervey JP (1982) Neuronal firing in the inferotemporal cortex of the monkey in a visual memory task. J Neurosci 2:361-375.

Gothard KM, Battaglia FP, Erickson CA, Spitler KM, Amaral DG (2007) Neural responses to facial expression and face identity in the monkey amygdala. J Neurophysiol 97:1671-1683.

Grill-Spector K, Malach R (2004) The human visual cortex. Annu Rev Neurosci 27:649-677.

Gross CG, Rocha-Miranda CE, Bender DB (1972) Visual properties of neurons in inferotemporal cortex of the Macaque. J Neurophysiol 35:96-111.

Hanes DP, Thompson KG, Schall JD (1995) Relationship of presaccadic activity in frontal eye field and supplementary eye field to saccade initiation in macaque: Poisson spike train analysis. Exp Brain Res 103:85-96.

Hasselmo ME, Rolls ET, Baylis GC (1989) The role of expression and identity in the face-selective responses of neurons in the temporal visual cortex of the monkey. Behav Brain Res 32:203-218.

Hung CP, Kreiman G, Poggio T, DiCarlo JJ (2005) Fast readout of object identity from macaque inferior temporal cortex. Science 310:863-866.

Insausti R, Juottonen K, Soininen H, Insausti AM, Partanen K, Vainio P, Laakso MP, Pitkänen A (1998) MR volumetric analysis of the human entorhinal, perirhinal, and temporopolar cortices. AJNR Am J Neuroradiol 19:659-671.

Keysers C, Xiao DK, Földiák P, Perrett DI (2001) The speed of sight. J Cogn Neurosci 13:90-101.

Kiani R, Esteky H, Tanaka K (2005) Differences in onset latency of macaque inferotemporal neural responses to primate and non-primate faces. J Neurophysiol 94:1587-1596.

Kirchner H, Thorpe SJ (2006) Ultra-rapid object detection with saccadic eye movements: visual processing speed revisited. Vision Res 46:1762-1776.

Kreiman G, Koch C, Fried I (2000) Category-specific visual responses of
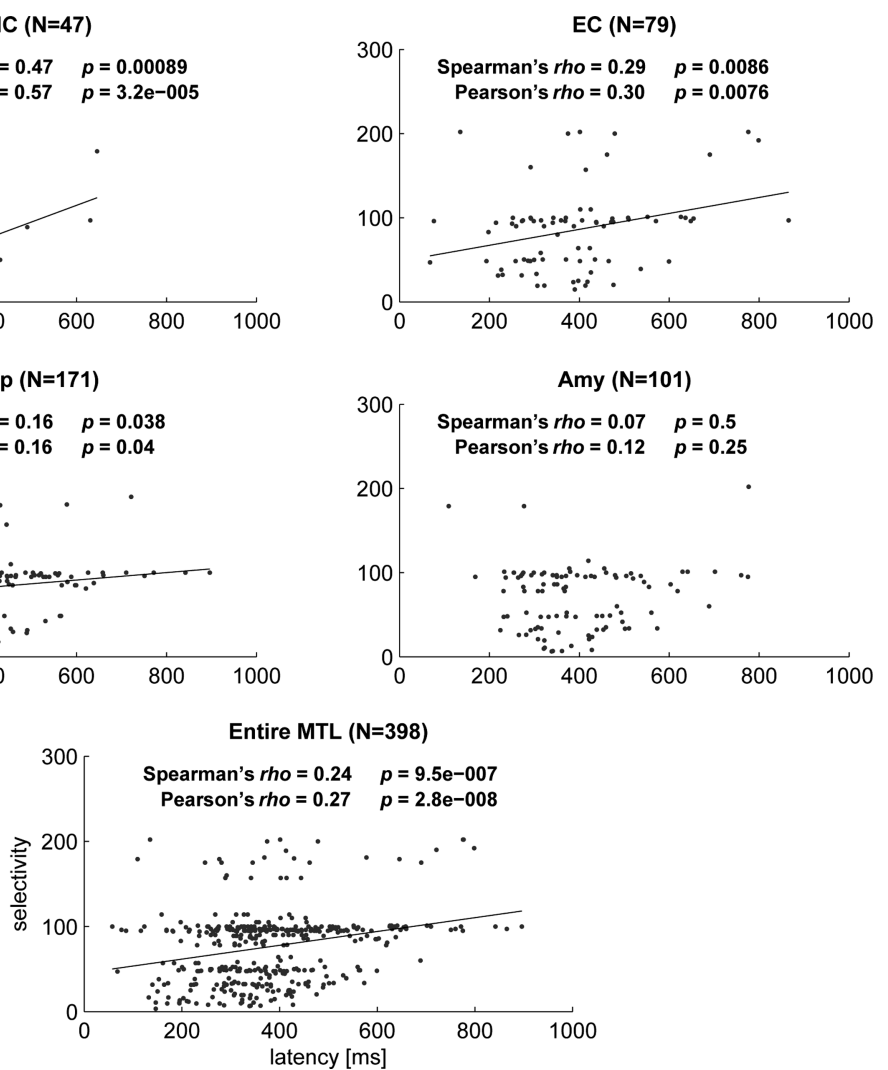

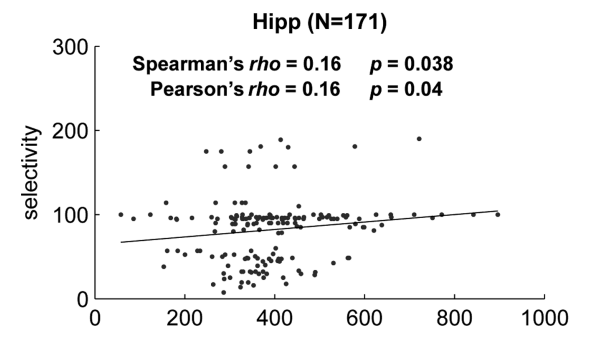

Scattergrams displaying selectivity versus latency for the four MTL regions and the entire MTL. Parametric (Pearas the reciprocal value of the percentage of stimuli to which a given cell responded. Note that the horizontal band round a selectivity value of 100 represents cells that responded to only one stimulus in sessions in which $\sim 100$ stimuli were shown. PHC, Parahippocampal cortex; EC, entorhinal cortex; Hipp, hippocampus; Amy, amygdala.

single neurons in the human medial temporal lobe. Nat Neurosci 3:946-953.

Leonard CM, Rolls ET, Wilson FA, Baylis GC (1985) Neurons in the amygdala of the monkey with responses selective for faces. Behav Brain Res 15:159-176.

Li L, Miller EK, Desimone R (1993) The representation of stimulus familiarity in anterior inferior temporal cortex. J Neurophysiol 69:1918-1929.

Liu Z, Richmond BJ (2000) Response differences in monkey TE and perirhinal cortex: stimulus association related to reward schedules. J Neurophysiol 83:1677-1692.

Logothetis NK, Sheinberg DL (1996) Visual object recognition. Annu Rev Neurosci 19:577-621.

Nakamura K, Mikami A, Kubota K (1992) Activity of single neurons in the monkey amygdala during performance of a visual discrimination task. J Neurophysiol 67:1447-1463.

Nakamura K, Matsumoto K, Mikami A, Kubota K (1994) Visual response properties of single neurons in the temporal pole of behaving monkeys. J Neurophysiol 71:1206-1221.

Naya Y, Yoshida M, Miyashita Y (2001) Backward spreading of memoryretrieval signal in the primate temporal cortex. Science 291:661-664.

Naya Y, Yoshida M, Miyashita Y (2003) Forward processing of long-term associative memory in monkey inferotemporal cortex. J Neurosci 23:2861-2871.

Ohman A, Carlsson K, Lundqvist D, Ingvar M (2007) On the unconscious subcortical origin of human fear. Physiol Behav 92:180-185.

Oram MW, Perrett DI (1992) Time course of neural responses discriminating different views of the face and head. J Neurophysiol 68:70-84.

Perez-Orive J, Mazor O, Turner GC, Cassenaer S, Wilson RI, Laurent G (2002) Oscillations and sparsening of odor representations in the mushroom body. Science 297:359-365.

Perrett DI, Rolls ET, Caan W (1982) Visual neurones responsive to faces in the monkey temporal cortex. Exp Brain Res 47:329-342. 
Perrett DI, Smith PA, Potter DD, Mistlin AJ, Head AS, Milner AD, Jeeves MA (1984) Neurones responsive to faces in the temporal cortex: studies of functional organization, sensitivity to identity and relation to perception. Hum Neurobiol 3:197-208.

Perrett DI, Mistlin AJ, Chitty AJ, Smith PA, Potter DD, Broennimann R, Harries M (1988) Specialized face processing and hemispheric asymmetry in man and monkey: evidence from single unit and reaction time studies. Behav Brain Res 29:245-258.

Pitkänen A, Kelly JL, Amaral DG (2002) Projections from the lateral, basal, and accessory basal nuclei of the amygdala to the entorhinal cortex in the macaque monkey. Hippocampus 12:186-205.

Quiroga RQ, Nadasdy Z, Ben-Shaul Y (2004) Unsupervised spike detection and sorting with wavelets and superparamagnetic clustering. Neural Comput 16:1661-1687.

Quiroga RQ, Reddy L, Kreiman G, Koch C, Fried I (2005) Invariant visual representation by single neurons in the human brain. Nature 435:1102-1107.

Quiroga RQ, Reddy L, Koch C, Fried I (2007) Decoding visual inputs from multiple neurons in the human temporal lobe. J Neurophysiol 98:1997-2007.

Quiroga RQ, Kreiman G, Koch C, Fried I (2008) Sparse but not "grandmother-cell" coding in the medial temporal lobe. Trends Cogn Sci 12:87-91.

Riches IP, Wilson FA, Brown MW (1991) The effects of visual stimulation and memory on neurons of the hippocampal formation and the neighboring parahippocampal gyrus and inferior temporal cortex of the primate. J Neurosci 11:1763-1779.

Rodland EA (2006) Simes' procedure is "valid on average." Biometrika 93:742-746

Rolls ET, Miyashita Y, Cahusac PM, Kesner RP, Niki H, Feigenbaum JD, Bach L (1989) Hippocampal neurons in the monkey with activity related to the place in which a stimulus is shown. J Neurosci 9:1835-1845.

Rolls ET, Cahusac PM, Feigenbaum JD, Miyashita Y (1993) Responses of single neurons in the hippocampus of the macaque related to recognition memory. Exp Brain Res 93:299-306.

Rolls ET, Xiang J, Franco L (2005) Object, space, and object-space representations in the primate hippocampus. J Neurophysiol 94:833-844.

Rutishauser U, Mamelak AN, Schuman EM (2006) Single-trial learning of novel stimuli by individual neurons of the human hippocampusamygdala complex. Neuron 49:805-813.

Rutishauser U, Schuman EM, Mamelak AN (2008) Activity of human hip- pocampal and amygdala neurons during retrieval of declarative memories. Proc Natl Acad Sci U S A 105:329-334.

Sanghera MK, Rolls ET, Roper-Hall A (1979) Visual responses of neurons in the dorsolateral amygdala of the alert monkey. Exp Neurol 63:610-626.

Sheinberg DL, Logothetis NK (2001) Noticing familiar objects in real world scenes: the role of temporal cortical neurons in natural vision. J Neurosci 21:1340-1350.

Somogyi P, Klausberger T (2005) Defined types of cortical interneurone structure space and spike timing in the hippocampus. J Physiol 562:9-26.

Squire LR, Stark CE, Clark RE (2004) The medial temporal lobe. Annu Rev Neurosci 27:279-306.

Suzuki WA (1996) Neuroanatomy of the monkey entorhinal, perirhinal and parahippocampal cortices: organization of cortical inputs and interconnections with amygdala and striatum. Semin Neurosci 8:3-12.

Suzuki WA, Amaral DG (1994) Perirhinal and parahippocampal cortices of the macaque monkey: cortical afferents. J Comp Neurol 350:497-533.

Suzuki WA, Miller EK, Desimone R (1997) Object and place memory in the macaque entorhinal cortex. J Neurophysiol 78:1062-1081.

Tamura H, Tanaka K (2001) Visual response properties of cells in the ventral and dorsal parts of the macaque inferotemporal cortex. Cereb Cortex 11:384-399.

Tanaka K (1996) Inferotemporal cortex and object vision. Annu Rev Neurosci 19:109-139.

Tovee MJ, Rolls ET, Azzopardi P (1994) Translation invariance in the responses to faces of single neurons in the temporal visual cortical areas of the alert macaque. J Neurophysiol 72:1049-1060.

Watanabe T, Niki H (1985) Hippocampal unit activity and delayed response in the monkey. Brain Res 325:241-254.

Waydo S, Kraskov A, Quian Quiroga R, Fried I, Koch C (2006) Sparse representation in the human medial temporal lobe. J Neurosci 26:10232-10234.

Wilson FA, Riches IP, Brown MW (1990) Hippocampus and medial temporal cortex: neuronal activity related to behavioural responses during the performance of memory tasks by primates. Behav Brain Res 40:7-28.

Xiang JZ, Brown MW (1998) Differential neuronal encoding of novelty, familiarity and recency in regions of the anterior temporal lobe. Neuropharmacology 37:657-676.

Xiang JZ, Brown MW (1999) Differential neuronal responsiveness in primate perirhinal cortex and hippocampal formation during performance of a conditional visual discrimination task. Eur J Neurosci 11:3715-3724.

Yanike M, Wirth S, Suzuki WA (2004) Representation of well-learned information in the monkey hippocampus. Neuron 42:477-487 . 